\title{
Early Detection Branch
}

National Cancer Institute

\section{Source}

National Cancer Institute. Early Detection Branch. NCI Thesaurus. Code C19520.

Development of scientific information and concepts, and dissemination of knowledge regarding early detection techniques, practices, and strategies to reduce mortality and morbidity from cancer. The program supports clinical trials and other appropriate research, fosters technological development, and encourages the publication of scientific findings and adoption of early detection practices. 